\title{
BMJ Open An investigation into the role of alcohol in self-harm in rural Sri Lanka: a protocol for a multimethod, qualitative study
}

\author{
Jane Brandt Sørensen, ${ }^{1}$ Thilde Rheinländer, ${ }^{1}$ Birgitte Refslund Sørensen, ${ }^{2}$ \\ Melissa Pearson, ${ }^{3}$ Thilini Agampodi, ${ }^{4}$ Sisira Siribaddana, ${ }^{5}$ Flemming Konradsen ${ }^{1}$
}

To cite: Sørensen JB, Rheinländer T, Sørensen BR, et al. An investigation into the role of alcohol in self-harm in rural Sri Lanka: a protocol for a multimethod, qualitative study. BMJ Open 2014;4: e005860. doi:10.1136/ bmjopen-2014-005860

- Prepublication history for this paper is available online. To view these files please visit the journal online (http://dx.doi.org/10.1136/ bmjopen-2014-005860).

CrossMark

For numbered affiliations see end of article.

Correspondence to Jane Brandt Sørensen; janebs@sund.ku.dk

\section{ABSTRACT}

Introduction: Sri Lanka has one of the highest suicide and self-harm rates in the world and although alcohol has been found to be a risk factor for self-harm in Sri Lanka, we know little about the connection between the two. This paper comprises a protocol for a qualitative study investigating alcohol's role in selfharm in rural Sri Lanka at three levels: the individual, community and policy level. The analysis will bring new understanding of the link between alcohol and self-harm in Sri Lanka, drawing on structural, cultural and social concepts. It will equip researchers, health systems and policy makers with vital information for developing strategies to address alcohol-related problems as they relate to self-harm.

Methods and analysis: To capture the complexity of the link between alcohol and self-harm in the Anuradhapura district in the North Central Province in Sri Lanka, qualitative methods will be utilised. Specifically, the data will consist of serial narrative life-story interviews with up to 20 individuals who have non-fatally selfharmed and where alcohol directly or indirectly was involved in the incidence as well as with their significant others; observations in communities and families; six focus group discussions with community members; and key-informant interviews with 15-25 stakeholders who have a stake in alcohol distribution, marketing, policies, prevention and treatment as they relate to self-harm.

Ethics and dissemination: The study has received ethical approval from the Ethical Review Committee of the Faculty of Medicine and Allied Sciences, Rajarata University of Sri Lanka. A sensitive data collection technique will be used and ethical issues will be considered throughout the study.

Results: The results will be disseminated in scientific peer-reviewed articles in collaboration with Sri Lankan and other international research partners.

\section{INTRODUCTION}

Deliberate self-harm and suicide is a serious social, economic and public health issue, being one of the top 20 leading causes of death globally. ${ }^{1}$ The issue is particularly pronounced in Asia, where $60 \%$ of suicides

\section{Strengths and limitations of this study}

- The study will provide new knowledge and an increased understanding of an area that has previously received limited research attention-the structural, cultural and social context behind the link between alcohol and self-harm in rural Sri Lanka.

- Quality of the information gathered and comprehensiveness are ensured by collecting data at several levels and through triangulation between data sources, informant groups and theories.

- One limitation of the study is the sensitivity of the issues of alcohol and self-harm, which may limit participation in the study.

- Owing to the nature of the methodologies utilised, the results require further studies in order to be transferable to other settings.

occur. $^{2}$ Although a decrease has been seen in recent years (from 47/100 000 population in $1995^{3}$ to $28.8 / 100000$ in 2012 - the latter estimate is based on expert modelling methods), ${ }^{4}$ Sri Lanka still has one of the highest suicide and self-harm rates globally. ${ }^{5}$ It has been found that problematic alcohol use is often involved in cases of self-harmeither in the person who consumes the alcohol or in someone affected by it. ${ }^{5-11}$ With a qualitative approach, this study will explore the structural, social and cultural behaviour surrounding alcohol's role in selfharm at the individual, community and policy level in the Anuradhapura district in the North Central Province in Sri Lanka.

The objectives of this study are to:

1. Investigate the explanatory models and coping strategies for alcohol use with a focus on its relation with self-harm in households in rural Sri Lanka;

2. Investigate community perceptions and responses to alcohol use and its relation with self-harm in rural Sri Lankan communities; 
3. Investigate stakeholders' involvement, influence on and motivations in controlling harmful effects of alcohol in Sri Lanka with a special focus on preventing self-harm.

\section{BACKGROUND}

\section{Alcohol, self-harm and suicide}

International research shows that acute and chronic alcohol use are both associated with suicidal behaviour, ${ }^{12-17}$ influenced by access to alcohol in a certain context ${ }^{15}$ as well as by the amounts consumed. ${ }^{18}$ It is well established that alcohol misuse is not merely an individual or psychological phenomenon, but a sociological one that has a profound impact on deviations found in suicidal behaviour. ${ }^{14}{ }^{19}$ The relationship between alcohol and self-harm is complex and seems to vary between genders, cultures and countries. ${ }^{15} 2021$ Problematic alcohol use affects social and family life, and poses risks not only for individual dysfunction, but also for relational conflict and family breakdown. ${ }^{15}$ Alcohol often acts as a long-term risk factor for selfharm, for example, through social and financial problems, domestic violence and effects on mood. ${ }^{22}$ However, in the short-term, it may have an acute level effect on the mood and increase the risk for impulsive and destructive behaviour even when misused by individuals without a chronic alcohol problem. ${ }^{20}$ Self-harm or suicide may thus be an end point for a long-term or short-term drinker, but a significant other of an alcohol misuser may also deliberately self-harm, in seeking to escape the negative influences of the alcohol. ${ }^{21} 23$

\section{Alcohol, self-harm and suicide in Sri Lanka}

It should be noted that the term 'self-harm' is used throughout this paper to describe deliberate injury to oneself. This definition captures two important points; that most cases of self-harm in the Sri Lankan context are non-fatal and with little or no intent to die, and can thus not be classified as 'suicide attempts'. ${ }^{24}$

Even though extensive international literature on the relationship between alcohol and self-harm exists, there has been little emphasis on the cultural and behavioural aspects underlying the connection in Sri Lanka. A few researchers have touched on it $^{725-27}$ and a Sri Lankan study of 159 acute self-poisoning cases-the most common method of self-harm in Sri Lanka being pesticide poisoning ${ }^{3}$-found that $32 \%$ were visibly affected by alcohol when admitted to hospital, which was confirmed by family and bystanders. ${ }^{7}$ Another study conducted a psychological autopsy of 372 suicides in rural Sri Lanka to find that problem drinking or alcohol dependence was common among male suicides in $61 \%$ of cases while alcohol misuse in another family member contributed to $14 \%$ of female suicides. ${ }^{9}$ None of these studies thoroughly investigated the dynamics and complex interlinkages between alcohol and self-harm and researchers have called for further investigation of the links between alcohol, impulsive behaviour and selfharm in Sri Lanka. ${ }^{23}$

\section{Alcohol use in Sri Lanka}

Officially, alcohol consumption is relatively low in Sri Lanka with a per capita intake of $3.7 \mathrm{~L}$ in 2010 (2.2 in 2005), which includes the WHO estimate of unrecorded liquor. ${ }^{17}$ However, Sri Lankan alcohol consumption data is believed to be inaccurate. ${ }^{28}$ Most alcohol consumed is the easily accessible, cheap, unrecorded and illicitly brewed 'kassipu', mainly consumed in poor, rural areas, ${ }^{25} 2930$-and difficult to document. Some suggest that up to $50-90 \%$ of alcohol consumed is illicit 263031 and the actual alcohol consumption level potentially much higher. Further, with a lifetime abstinence rate at a high $69 \%,{ }^{17}$ many drinkers consume alcohol above the average.

Alcohol consumption in Sri Lanka is almost exclusively a male practise ${ }^{27}$ (annual per capita consumption in 2010 was $7.3 \mathrm{~L}$ for men and $0.3 \mathrm{~L}$ for women), ${ }^{17}$ and the types of alcohol consumed are closely linked to class and status. ${ }^{31}$ Alcohol intoxication in Sri Lanka has primarily been used as a means to reduce tension, to a moderate degree at ceremonies and less so for personal enjoyment. ${ }^{26}{ }^{30-32}$ It predominately takes place for the sole purpose of intoxication. ${ }^{30}{ }^{33}$ A Sri Lankan study of individuals who self-harmed in rural areas found that they regarded drinking as a reasonable response to the stresses of being a farmer. ${ }^{7}$ In addition, a selfadministered questionnaire among young Sri Lankan men found that alcohol was used to dominate surroundings and to become more prominent among peers. ${ }^{32}$ However, much remains to be understood about how these motives for alcohol use are linked to cases of self-harm.

\section{Alcohol and its effects on family and social life}

Sri Lankan research shows that social harm from alcohol contributes to violence ${ }^{27}$ and domestic violence ${ }^{34}$; impacts the family budget and duties; brings about feelings of shame and guilt ${ }^{35-37}$; and instigates self-harm. ${ }^{7} \mathrm{~A}$ UNICEF study among Sri Lankan adolescents found that $18.3 \%$ of their fathers drank alcohol and $26.6 \%$ of them became violent afterwards. ${ }^{38}$ It also seems that selfharm by women and children is often linked to a husband or father's drinking. ${ }^{27}$ Such consequences of excessive alcohol use and how it affects family and social life will be investigated in this study to be able to understand the connection between alcohol and self-harm.

\section{Alcohol and self-harm at the community level}

Community members' attitudes towards alcohol use may affect how alcohol users, families and communities interact. $^{39}$ Societal stigmatisation and norms may have a negative influence on the individual and family members ${ }^{26}$ but, at the same time, the community can play a role in supporting members who have problems related to alcohol use and in cases of self-harm or 
suicide. This link between the individual and the perceptions of the close social surroundings will be investigated in terms of how group concepts of alcohol norms, shame and support are relevant for the rural, Sri Lankan setting.

\section{Stakeholders in alcohol and self-harm prevention and interventions}

In Sri Lanka, a number of risk factors of self-harm have been addressed by limiting access to the means of selfharm or improving medical management of poisoning through regulations and ban of highly toxic pesticides used for self-poisoning. ${ }^{40}$ Although some ad hoc initiatives have been implemented responding to the issue of alcohol, including through taxes and policies (ie, ban of alcohol commercials at sports events) ${ }^{41}$; treatment (a Non-Government Organisation (NGO) treatment programme for alcohol abusers in Colombo) ${ }^{42}$; advocacy programmes on the prevention of alcohol misuse ${ }^{43}$; and NGO-led educational programmes, ${ }^{44} 45$ there is no thorough evaluation of their effectiveness and alcohol continues to be a major risk factor for self-harm in Sri Lanka. An investigation of the environment in which initiatives are developed is therefore timely.

\section{METHODS AND ANALYSIS}

\section{Conceptual framework}

The conceptual framework outlined below will guide the analytical process of the study. It builds on the recommendations of Hunt and Barker ${ }^{46}$ for carrying out sociocultural and anthropological research on drugs and alcohol.

\section{Local alcohol and self-harm terminologies}

Hunt and Barker ${ }^{46}$ urge researchers to examine how societies categorise alcohol use since there is no single definition of 'normal' drinking, problem drinking, misuse or alcohol dependence that applies equally to all cultures and environments. ${ }^{47}$ An open and investigatory approach to the topic will therefore be sought in this study when interacting with individuals and communities, ensuring that they explain their understanding of terms such as alcohol misuse, problem drinking, alcohol-related self-harm and harm to others.

\section{Relational aspects of alcohol and self-harm}

Hunt and Barker ${ }^{46}$ emphasise that alcohol is a social, relational and active phenomenon, and alcohol problems and solutions should be seen in this light. It is therefore central to this study to investigate and understand more of how damage or social harm from the use of alcohol affects not only the drinker, but the close social relations and environment of the drinker. ${ }^{39} 4849$

\section{Power relations and culturally accepted behaviour}

Finally, the authors highlight that alcohol consumption is closely linked to power relationships in terms of accepted behaviours in certain social and cultural contexts, including how different social groups or classes fashion and control acceptability of alcohol use and how production and distribution are organised. ${ }^{46}$ As part of this study, power relations and alcohol cultures at community levels and alcohol consumption patterns for different social classes will be explored. Additionally, national alcohol policies, production and prevention will also be investigated, thus adding more layers to the analysis of alcohol cultures and power dynamics.

\section{Explanatory models}

The mentioned concepts will be investigated through the overall theory of Kleinmann's 'explanatory models, ${ }^{50}$ examining how illnesses are understood and dealt with through ethnocultural lenses,${ }^{51}$ that is, how individuals and communities make sense of illness, health and suffering. ${ }^{50}$ More specifically, explanatory models will be used to investigate how people explain the use of alcohol in relation to self-harm.

\section{Coping with life, alcohol and self-harm}

In addition to the concepts drawn from Hunt and Barker, 'coping' is central to this study.

According to Lazarus, coping is defined as the way individuals interact with stressors when trying to return to a 'normal' functioning level after a stressful situation $^{52}$ and how they manage by minimising, changing or accepting it. ${ }^{53}$ The process of coping is complex and many factors play a role in how alcohol and/or self-harm are coped with or how the behaviours act as actual coping strategies. In fact, alcohol use has been described as a concrete coping mechanism to get through a tough time for farmers in Sri Lanka, ${ }^{7}$ while family members at the same time have to cope with the consequences of the alcohol use or the possible subsequent self-harm. Self-harm has also been described as a form of coping, which can be used to change an unwanted situation. ${ }^{24}$ These processes and mechanisms of coping will be investigated to understand the dynamics and strategies applied by drinkers and their families in cases of alcohol-related self-harm.

\section{Study setting}

The study will mainly be carried out in the Anuradhapura district in the North Central Province, where one of the highest suicide rates in Sri Lanka persists. ${ }^{54}$ It is connected to the 'Safe Storage' study, which is a large community-based, cluster randomised control trial evaluating the effectiveness in storing pesticides safely to reduce self-harm. ${ }^{55}$ In the safe storage study, 795 cases of self-harm were identified among individuals admitted to Anuradhapura Teaching Hospital and 11 peripheral hospitals in a year (2013-2014).

\section{Study design}

Extensive quantitative research has been carried out on the topic of self-harm ${ }^{56-58}$ and alcohol ${ }^{35}{ }^{59}$ separately in 
Sri Lanka, however, limited qualitative research has been conducted. ${ }^{266061}$ This study responds to this gap by utilising a qualitative research approach, seeking an in-depth perspective of the individual's and communities' interpretations and perceptions of these phenomena. A range of qualitative methods will be applied: observations; narrative, life-story interviews; focus group discussions (FGDs); and semistructured, stakeholder interviews.

\section{Inclusion criteria and selection of participants}

The first part of the study will include individuals who non-fatally self-harmed and where alcohol played a role in the incident. Either (A) the individual was under the influence of alcohol when admitted to hospital (7-10 cases); or (B) the incident was sparked by another's alcohol consumption (7-10 cases). We are aware that by choosing this selection strategy, we exclude those who for different reasons did not get admitted to hospital as well as those who fatally self-harmed.

Participants will be sampled through three peripheral hospitals, representing different socioeconomic characteristics, agricultural production and history of settlement. ${ }^{57}$ The majority of serious self-harm cases from these three hospitals are transferred to Anuradhapura Teaching Hospital and by permission from the Hospital Director, cases being admitted from the three hospitals will be sampled from here. (A) and (B) will be identified by trained Safe Storage research officers, who are already on a daily basis identifying all self-harm cases within the study area. Mechanisms for standard reporting and measures of quality insurance have been put into place and their presence are supported at the Province and District levels.

At the hospital, possible participants will be asked for oral as well as written consent for the study team to contact them a minimum of 1 week after they are discharged, at which point informed consent will be repeated and reobtained.

After interviewing (A) and (B), they will be asked for consent for the team to talk with their (C) significant other(s). Written and oral consent will also be sought from this group.

Approximately 15 cases of (A), (B) and (C) will be interviewed several times (approximately up to three times) and visited for observational sessions, depending on consent from the whole household.

In order to obtain a comprehensive understanding of communities' perceptions of alcohol use and how it relates to self-harm, FGDs will be conducted. Participants (D) for the FGDs will be selected through snowballing among community members and represent homogenous groups of male and female adults from the selected communities. Participants will not necessarily be from households where alcohol use or self-harm is an issue.

Finally, to examine the environment in which alcohol and self-harm prevention and treatment initiatives, regulations and policies have and will be developed, a stakeholder-analysis will be carried out. The participants (E) will be purposively selected from previous data collection and research, as well as through snowballing. New key informants will be added until no more relevant stakeholders can be identified and saturation is reached.

\section{Narrative life-story interviews}

In-depth interviews are appropriate when seeking to obtain meaning ${ }^{62}$ and get people's own perspective of a situation. ${ }^{63}$ For the first objective, the interviews will have a narrative life-story component in order to obtain an understanding of the individual's life, along with the events and decisions that led up to the self-harm incidence. Themes to be explored include: perceptions and beliefs of alcohol use and misuse; alcohol preferences; availability and access to alcohol; perceived impact of alcohol use; coping strategies used by alcohol consumers and significant others; and explanatory models of selfharm, including perceived causes. If deemed relevant a timeline will be made in collaboration with the participant to make a visual overview of important events, which can be helpful in encouraging participants to talk while reversing the power-roles between the researcher and the participant. ${ }^{64}$

The narrative life-story interviews will be carried out in face-to-face meetings with (A), (B) and (C) in a quiet place, that is, in their homes or in another relevant setting, as determined by the participants. The researchers will engage with the participants through a series of interviews to construct a comprehensive life-story narrative.

\section{Participant observations}

Observations will be key in gaining an in-depth understanding of the local alcohol culture, social structures and interactions. It can help to showcase implicit features of social life, provide context of behaviour ${ }^{63}$ and shed light on non-explicit knowledge. ${ }^{65}$ Observations will take place during daily life in selected families observing dynamics; power relations; alcohol consumption in terms of who consumes, when, how much, and what; and strategies employed by family members in regard to alcohol use and previous self-harm. Observations in communities will include observing social organisation of communities and people; alcohol selling establishments and how selling and consumption is organised; how alcohol is talked about; and how people react towards intoxicated people. During community observations, the research team will make sure to openly explain the purpose of the research to anyone enquiring and abstain from observing if community members feel disturbed or uncomfortable about the presence of the team.

\section{Focus group discussions}

FGDs are effective when seeking a broad range of opinions on a topic and they provide the opportunity for 
participants to probe each other's reasons for holding a specific view. ${ }^{63}$ Knowing that this method will bring out group norms of "what should be, ${ }^{62}$ discussion themes will be verbalised as general, non-personal issues. Topics will include general perceptions of alcohol use; reactions towards people who consume alcohol; explanations for use of alcohol; how alcohol is associated with self-harm; and how the community responds to alcohol use and self-harm. In addition, small vignettes covering different scenarios of alcohol in connection with self-harm will be used to spur conversation. Vignettes are particularly useful when exploring sensitive topics, as they can help distance participants from the topic of discussion, ${ }^{66}$ which has proven beneficial in other studies on selfharm carried out in Sri Lanka. ${ }^{7}$

Approximately six FGDs will be carried out. Since previous research in Sri Lanka has shown gender differences in alcohol intake and perceptions toward alcohol use, ${ }^{7}$ they will be held in age and gender-segregated groups, with a maximum of 10 participants in each group.

\section{Stakeholder interviews}

The stakeholder analysis will follow three main steps as outlined by Varvasovszky and Brugha: (1) identifying and approaching stakeholders; (2) describing stakeholder's positions, responsibilities and collaborations; and (3) diagnosing and suggesting strategies for future interventions. ${ }^{67}$ Semistructured interviews will be conducted with 15-25 stakeholders who have a stake in the decision making and implementation process ${ }^{68}$ related to alcohol and self-harm policies and interventions in Sri Lanka. Some of the themes to be explored include activities carried out on preventing alcohol misuse and/ or self-harm; interests in and agendas for prevention of self-harm and controlling of alcohol; means of influencing the agenda, including interrelations with other stakeholders in this area; and past, present and future perspectives.

Stakeholders will include representatives from different organisational layers:

- Producers and traders of alcohol;

- Community representatives (leaders, priests, teachers);

- NGOs (Alcohol and Drug Information Centre (ADIC), Mel Medura, Sumithrayo, The Foundation for Innovative Social Development (FISD) and others);

- Academia;

- The health system (public, private and ayurvedic sectors at several levels);

- Government (from ministries of Health; Education; Consumer Welfare; Child Development and Women's Affairs; the National Child Protection Authority and others).

\section{Data management}

All interview guides will be translated from English to Sinhala by trained translators and validated by representatives from the larger research group. The interview and FGD guides will be pilot tested in similar informant groups and revised accordingly. Interviews and FGDs will be conducted and facilitated by English/Sinhala speaking research assistants, supported by the first author. All interviews and FGDs will be audio recorded with the informed consent of all participants. The research assistants will carry out verbatim Sinhala transcriptions of all interviews and FGDs, which will then be translated into English. Field notes will be taken at all occasions. Observational data will be captured in extensive descriptive field notes and analytical memos, which will be transferred to a software programme on a daily basis and discussed within the research team. These discussions and reflections will be added to the observational notes and serve as important tentative steps of the analysis.

\section{Analysis}

Analysis will be inductive and dynamic, starting immediately after the first interviews, FGDs and observations, continuing until the phenomena under study are well understood. It will be informed by the described conceptual framework and rely on a continuous triangulation between the emerging analytical and the predefined theoretical concepts in conjunction with the diverse sources of empirical data. The analysis will consequently not be entirely predefined, but reliant on the emerging explanatory models and understandings of alcohol's role in selfharm. The analysis of interview and FGD data will follow the principles of systematic content analysis ${ }^{63}$ : The transcripts of the interviews and FGDs will be coded to identify themes, ideas and patterns, and these codes will be grouped into broader themes. During the analysis, the English version will be controlled and compared with the Sinhala transcriptions, in order to ensure inclusion of all relevant context and local matters in the analysis. ${ }^{69}$

\section{ETHICS AND DISSEMINATION}

\section{Informed consent}

Verbal and written information about the aim of the study will be given in English or Sinhala, as appropriate. In each case, the capacity of the participant to give consent will be assessed-for example, a highly intoxicated person will not be able to make an informed decision. Participants will be informed that they can withdraw from the study at any time and that participation-or lack of it-will not have negative consequences for them. For the serial, narrative life-story interviews and observations, the continued participation will be verbally reconfirmed at each visit. Participants will be informed that all data collected will be stored safely and only used by researchers, maintaining confidentiality.

\section{Validity}

To validate the findings of this study, a long period of field work (minimum 10 months) has been allotted. This extensive time allows for a comprehensive 
collection of multiple sources of data and an in-depth understanding of the phenomenon under study. ${ }^{69} \mathrm{~A}$ rigorous transcription process with external cross-checks will ensure highest data quality and consistency in definitions of codes and analytical themes. ${ }^{69}$ Discrepant information running counter to the themes will also be presented. $^{70}$ The use of triangulation at several levels as well as detailed descriptions of the contextual setting of the study will enhance the comprehensiveness of the findings. ${ }^{71}$ Finally, coresearchers from different research disciplines will be engaged to discuss the interpretations of the study to ensure that the conclusions resonate with the broader research community. ${ }^{71}$

\section{Reflexivity}

In qualitative research, the researchers' background, culture and experiences influence the interpretation of the data. ${ }^{69}$ According to Malterud, ${ }^{72}$ these preconceived ideas are a necessary precondition for understanding a topic and reflexivity starts by identifying such preconceptions brought into the project by the researchers. This will carefully be considered throughout the study by discussing such preconceptions within the research team, by maintaining diaries to systematically document and thus enhance trustworthiness and transparency. ${ }^{70}$

\section{Power dynamics}

In carrying out qualitative research it is important to be aware of power imbalances between the researcher and the participant, since the former can maintain control and lead the participants in a certain direction. Especially, the individuals in group (A), (B) and (C) are at some level vulnerable and marginalised. The research team will comprise local and international researchers, who in different ways differ from participants, that is, in terms of sex, socioeconomic status and age. Some of these imbalances can be decreased by building trust and avoiding leading questions. ${ }^{69}$ Further, the narrative, life-story approach has been chosen to empower the participant to lead the conversation, thereby shifting control.

\section{Ethical considerations}

The Helsinki Declaration of 'Good Clinical Practice' will guide the study design and ethical issues will be considered from the outset and throughout the study. Inquiring into other's personal life can be experienced as traumatic, which will be countered by utilising a sensitive data collection technique. While interviews about previous self-harm impacted participants' feelings, research indicated that the majority felt better afterwards $^{73}$ and a study involving family members of individuals who died from suicide showed that sensibly conducted interviews can have a valuable therapeutic effect. $^{74}$ However, the interview situation may still be upsetting and protecting the individual is a priority; that is, the interviews will be carried out alone, in a setting the participant prefers. To assess the participant's mood and whether the interview has influenced them, a Visual Analogue Scale (VAS) will be administered in which participants rate their mood before and after the interview. This method has been used in similar settings ${ }^{75}$ and will assist in identifying individuals who may be at increased risk of repeated self-harm. These participants will be offered more comprehensive support after the interview. Irrespective of the rating on the VAS the importance of seeking support when feeling distressed will be highlighted and all participants will be given information about where to seek help.

\section{Dissemination}

At the end of the study, findings will be communicated to the community and disseminated in peer-reviewed, internationally recognised journals in collaboration with Sri Lankan and other partners with maximum visibility for Sri Lankan researchers. Articles will also be submitted to other types of media to increase awareness of the topic.

\section{Author affiliations}

${ }^{1}$ Department of International Health, Immunology and Microbiology, University of Copenhagen, Copenhagen, Denmark

${ }^{2}$ Department of Anthropology, University of Copenhagen, Copenhagen, Denmark

${ }^{3}$ Pharmacology, Toxicology and Therapeutics, University of Edinburgh, UK and South Asian Clinical Toxicology Research Collaboration, Edinburgh, UK ${ }^{4}$ Department of Community Medicine, Faculty of Medicine and Allied Sciences, Rajarata University of Sri Lanka, Saliyapura, Sri Lanka

${ }^{5}$ Department of Medicine, Faculty of Medicine and Allied Sciences, Rajarata University of Sri Lanka, Saliyapura, Sri Lanka

Acknowledgements The authors would like to thank the Safe Storage field staff, SACTRC Office staff and staff at Department of Community Medicine, Faculty of Medicine and Allied Sciences, Rajarata University of Sri Lanka for organisational support, especially Manjula Weerasinghe and Ravi Pieris for their assistance in setting up the study. The authors appreciate the guidance, helpful comments and support from Michael Eddleston, Andrew Dawson and Suneth Agampodi.

Contributors JBS drafted the initial manuscript and developed the protocol. TR and FK collaborated on conceptualising and designing the study and contributed to the draft of the manuscript. BRS provided valuable input to methodology and background information on the study site. MP contributed towards the study set-up, logistics and methodology. TA contributed to the context, cultural specificities, methodology and ethical considerations. SS furnished information on the structure of health systems, in addition to assisting with ethical and contextual considerations. All authors read and approved the final manuscript.

Competing interests None.

Ethics approval Ethical approval has been obtained from the Ethical Review Committee of the Faculty of Medicine and Allied Sciences, Rajarata University of Sri Lanka and the study has been reviewed by the Safe Storage research group.

Provenance and peer review Not commissioned; externally peer reviewed.

Open Access This is an Open Access article distributed in accordance with the Creative Commons Attribution Non Commercial (CC BY-NC 4.0) license, which permits others to distribute, remix, adapt, build upon this work noncommercially, and license their derivative works on different terms, provided the original work is properly cited and the use is non-commercial. See: http:// creativecommons.org/licenses/by-nc/4.0/ 


\section{REFERENCES}

1. World Health Organization. Mental health, suicide prevention. World Health Organization. 2014. http://www.who.int/mental health/ prevention/en/ (accessed May 2014).

2. Beautrais AL. Suicide in Asia. Crisis J Crisis Interv Suicide Prev 2006;27:55-7.

3. Gunnell D, Fernando R, Hewagama M, et al. The impact of pesticide regulations on suicide in Sri Lanka. Int $J$ Epidemiol 2007;36:1235-42.

4. World Health Organization. Preventing suicide, a global imperative World Health Organization. 2014. http://apps.who.int/iris/bitstream/ 10665/131056/1/9789241564779_eng.pdf?ua=1 (accessed Sep 2014).

5. Rajapakse T, Griffiths K, Christensen H. Characteristics of non-fatal self-poisoning in Sri Lanka: a systematic review. BMC Public Health 2013;13:331.

6. Van Der Hoek W, Konradsen F. Risk factors for acute pesticide poisoning in Sri Lanka. Trop Med Int Health 2005;10:589-96.

7. Konradsen F, Hoek W, Peiris P. Reaching for the bottle of pesticide -a cry for help. Self-inflicted poisonings in Sri Lanka. Soc Sci Med 2006;62:1710-19.

8. Eddleston M, Sudarshan K, Senthilkumaran M, et al. Patterns of hospital transfer for self-poisoned patients in rural Sri Lanka: implications for estimating the incidence of self-poisoning in the developing world. Bull World Health Organ 2006;84:276-82.

9. Abeyasinghe R, Gunnell D. Psychological autopsy study of suicide in three rural and semi-rural districts of Sri Lanka. Soc Psychiatry Psychiatr Epidemiol 2008;43:280-5.

10. Pearson M, Zwi AB, Rouse AK, et al. Taking stock-what is known about suicide in Sri Lanka: a systematic review of diverse literature. Crisis J Crisis Interv Suicide Prev 2014;1:1-12.

11. Samaraweera S, Sumathipala A, Siribaddana S, et al. Completed suicide among sinhalese in Sri Lanka: a psychological autopsy study. Suicide Life Threat Behav 2008;38:221-8.

12. World Health Organization. Public health action for the prevention of suicide-a framework. Geneva: World Health Organization, 2012.

13. Rehm J. The risks associated with alcohol use and alcoholism. Alcohol Res Health J Natl Inst Alcohol Abuse Alcohol 2011;34:135.

14. Cherpitel CJ, Borges GLG, Wilcox HC. Acute alcohol use and suicidal behavior: a review of the literature. Alcohol Clin Exp Res 2004;28:18S-28S.

15. Babor T, Caetano R, Casswell S, et al. Alcohol-no ordinary commodity. Research and public policy. 2nd edn. Oxford, New York: Oxford University Press, 2010

16. Kaplan MS, Huguet N, McFarland BH, et al. Use of alcohol before suicide in the United States. Ann Epidemiol 2014;24:588-92.e1-2.

17. World Health Organization. Global status report on alcohol and health, 2014. World Health Organization, 2014.

18. Borges G, Cherpitel C, Orozco R, et al. Multicentre study of acute alcohol use and non-fatal injuries: data from the WHO collaborative study on alcohol and injuries. Bull World Health Organ 2006;84:453-60.

19. Skog O-J. Alcohol and suicide-Durkheim revisited. Acta Sociol 1991;34:193-206.

20. Brady J. The association between alcohol misuse and suicida behaviour. Alcohol Alcohol 2006;41:473-8.

21. Rossow L. Alcohol and suicide-beyond the link at the individual level. Addiction 1996;91:1413-16.

22. Stephenson IM, Møller S. Alkoholmisbrug og selvmordsadfærd. Odense, Denmark: Center for Selvmordsforskning, 2003.

23. Rossow I, Moan IS. Parental intoxication and adolescent suicidal behavior. Arch Suicide Res 2012;16:73-84.

24. Marecek J, Senadheera C. "I drank it to put an end to me": narrating girls' suicide and self-harm in Sri Lanka. Contrib Indian Sociol 2012;46:53-82.

25. Jayasinghe NR, Foster JH. Deliberate self-harm/poisoning, suicide trends. The link to increased alcohol consumption in Sri Lanka. Arch Suicide Res 2011;15:223-37.

26. Gamburd MR. Breaking the ashes-the culture of illicit liquor in Sr Lanka. Ithaca, New York: Cornell University Press, 2008.

27. Marecek J. Young women's suicide in Sri Lanka: cultural, ecological, and psychological factors. Asian J Couns 2006;13:63-92.

28. Jayasekara RS, Schultz T. Health status, trends, and issues in Sri Lanka. Nurs Health Sci 2007;9:228-33.

29. Jaysinghe NRM, Foster JH. Acute poisoning and suicide/deliberate self-harm trends in Sri Lanka: alcohol a cause for concern-some further observations. Asian J Psychiatry 2010;3:233-4.

30. Baklien B, Samarasinghe D. Alcohol and poverty in Sri Lanka. FORUT Norway \& FORUT Sri Lanka; 2004. http://www. add-resources.org/alcohol-and-poverty-in-sri-lanka.444180-76584. html (accessed Jan 2014).

31. Abeyasinghe R. Illicit alcohol: drinking culture in Colombo. Colombo: Publisher Vijitha Yapa Co., 2002.

32. Perera $B$, Torabi M. Motivations for alcohol use among men aged 16-30 years in Sri Lanka. Int J Environ Res Public Health 2009;6:2408-16.

33. Abeyasinghe R. Towards an evidence based alcohol policy. Sri Lanka J Psychiatry 2011;2:1-2.

34. Subramaniam $\mathrm{P}$, Sivayogan $\mathrm{S}$. The prevalence and pattern of wife beating in the Trincomalee district in eastern Sri Lanka. Southeast Asian J Trop Med Public Health 2001;32:186-95.

35. Siriwardhana $\mathrm{P}$, Dawson $\mathrm{AH}$, Abeyasinge R. Acceptability and effect of a community-based alcohol education program in rural Sri Lanka. Alcohol Alcohol 2012;48:250-6.

36. Samarasinghe D. Sri Lanka: alcohol now and then. Addiction 2006;101:626-8.

37. Samarasinghe D. Treating alcohol problems in Sri Lanka. Br J Addict 1989;84:865-7.

38. Thalagala NI, Rajapakse L. National survey on emerging issues among adolescents in Sri Lanka. Colombo UNICEF. 2004. http:// www.shppsl.net/shn/site/wp-content/uploads/2011/12/ reproductive-health-studies-paper.pdf (accessed Jan 2014).

39. Kinney J. Loosening the grip: a handbook of alcohol information. 10th edn. New York: Mc Graw Hill, 2012.

40. Pearson M, Zwi AB, Buckley NA, et al. Policymaking "under the radar": a case study of pesticide regulation to prevent intentional poisoning in Sri Lanka. Health Policy Plan 2013. Published Online 20 Dec 2013.

41. National Authority on Tobacco and Alcohol. National Authority on Tobacco and Alcohol. 2009. http://www.nata.gov.lk (accessed Dec 2013)

42. Mel Medura. Mel Medura. 2013. http://melmedura.org/ (accessed Dec 2013)

43. Alcohol and Drug Information Center. Alcohol and Drug Information Center (ADIC Sri Lanka). 2009. http://www.adicsrilanka.org (accessed Dec 2013)

44. Healthy Lanka. Healthy Lanka. 2013. http://healthylanka.lk/ (accessed Dec 2013).

45. Foundation for Innovative Social Development. Foundation for innovative social development. 2013. http://www.fisd.lk/ (accessed Dec 2013).

46. Hunt G, Barker JC. Socio-cultural anthropology and alcohol and drug research: towards a unified theory. Soc Sci Med 2001;53:165-88.

47. Gusfield J. Contested meanings: the construction of alcohol problems. London: the University of Wisconsin Press, 1996.

48. Giesbrecht N, Cukier S, Steeves D. Collateral damage from alcohol: implications of "second-hand effects of drinking" for populations and health priorities. Addiction 2010;105:1323-5.

49. Casswell S, You RQ, Huckle T. Alcohol's harm to others: reduced wellbeing and health status for those with heavy drinkers in their lives. Addiction 2011;106:1087-94.

50. Kleinmann A, Eisenburg L, Good B. Culture, illness, and care: clinical lessons from anthropologic and cross-cultural research Ann Intern Med 1978;88:251-8.

51. Thompson T, Dorsey A, Miller K, et al. Handbook of Health Communication. New York \& London: Lawrence Erlbaum Associates, 2003

52. Ogden J. Health psychology - a text book. New York: Open University Press, 2004.

53. Lyon BL. Stress, coping and health. In: Lyon BL, ed. Handbook of Stress, Coping and Health - Implications for Nursing Research, Theory and Practice. Detroit, 2000:3-23.

54. Thalagala N. Suicide trends in Sri Lanka 1880-2006; social, demographic and geographical variations. J Coll Community Physicians Sri Lanka 2009;14:24-32.

55. Pearson M, Konradsen F, Gunnell D, et al. A community-based cluster randomised trial of safe storage to reduce pesticide self-poisoning in rural Sri Lanka: study protocol. BMC Public Health 2011;11:879-0.

56. Eddleston M, Gunnell D, Karunaratne A, et al. Epidemiology of intentional self-poisoning in rural Sri Lanka. Br J Psychiatry 2005;187:583-4.

57. Manuel C, Gunnell DJ, van der Hoek W, et al. Self-poisoning in rura Sri Lanka: small-area variations in incidence. BMC Public Health 2008;8:26

58. De Silva V, Hanwella R, Senanayake M. Age and sex specific suicide rates in Sri Lanka from 1995-2011. Sri Lanka J Psychiatry 2013;3:7-11. 
59. Samarasinghe DS, Dissanayake SAW, Wijesinghe CP. Alcoholism in Sri Lanka: an epidemiological survey. Br J Addict 1987;82:1149-53.

60. Widger T. Suffering, frustration, and anger: class, gender and history in Sri Lankan suicide stories. Cult Med Psychiatry 2012;36: 225-44.

61. Marecek J. Culture, gender and suicidal behaviour in Sri Lanka. Suicide Life-Threat Behav 1998;28:69-81.

62. Hardon A, Boonmongkon P, Streefland P, et al. Applied health research research manual-anthropology of health and health care. 3rd, revised edn. Amsterdam, The Netherlands: Het Spinhuis Publishers, 2001.

63. Bryman A. Social research methods. New York: Oxford University Press, 2008.

64. Mikkelsen B. Methods for development work and research. A guide for practitioners. New Delhi/Thousand Oaks/London: Sage Publications, 1995.

65. Baarts C. Håndværket_opbygning af viden. Ind $i$ Verden. 2nd ed. Denmark: Hans Reitzels Forlag, 2012.

66. Hughes R, Huby M. The application of vignettes in social and nursing research. J Adv Nurs 2002;37:382-6.

67. Varvasovszky Z, Brugha R. How to do (or not to do)... A stakeholder analysis. Health Policy Plan 2000;15:338-45.
68. Brugha R, Varvasovszky Z. Stakeholder analysis: a review. Health Policy Plan 2000;15:239-46.

69. Creswell J. Qualitative inquiry \& research design—choosing among five approaches. 3rd edn. Los Angeles, London, New Delhi, Singapore, Washington DC: Sage, 2013.

70. Reynolds J, Kizito J, Ezumah N, et al. Quality assurance of qualitative research: a review of the discourse. Health Res Policy Syst 2011;9:43.

71. Creswell J. Research design: qualitative, quantitative, and mixed methods approaches. 3rd edn. Los Angeles, London, New Delhi, Singapore: Sage, 2008.

72. Malterud K. Qualitative research: standards, challenges, and guidelines. Lancet 2001;358:483-8.

73. Biddle L, Cooper J, Owen-Smith A, et al. Qualitative interviewing with vulnerable populations: individuals' experiences of participating in suicide and self-harm based research. J Affect Disord 2013;145:356-62.

74. Owens $\mathrm{C}$, Owen $\mathrm{G}$, Lambert $\mathrm{H}$, et al. Public involvement in suicide prevention: understanding and strengthening lay responses to distress. BMC Public Health 2009;9:308.

75. Rivlin A, Marzano L, Hawton K, et al. Impact on prisoners of participating in research interviews related to near-lethal suicide attempts. J Affect Disord 2012;136:54-62. 